\title{
Comparative host genomics: how has human evolution affected our immune defence against hepatitis $C$ virus?
}

\author{
Connor GG Bamford*,1 \& John McLauchlan ${ }^{1}$ \\ ${ }^{1} \mathrm{MRC}$ - University of Glasgow Centre for Virus Research, 464 Bearsden Rd, Bearsden, Glasgow, G61 1QH, UK \\ *Author for correspondence: connor.bamford@glasgow.ac.uk
}
“'Intriguingly, while permissive for chronic HCV infection, rates of chronicity and associated disease differ in chimpanzees and treeshrews when compared with humans."

First draft submitted: 8 February 2019; Accepted for publication: 11 February 2019; Published online: 14 March 2019

Keywords: chimpanzee $\bullet$ evolution $\bullet$ genomics $\bullet$ HCV $\bullet$ hepatitis $C$ virus $\bullet$ host $\bullet$ human $\bullet$ immunity $\bullet$ interferon

Pathogens are undeniably a critical environmental pressure influencing the evolution of humans and other animals; the resulting genetic changes can significantly affect the outcome of an infection [1]. Fossil and genetic evidence suggests that anatomically modern humans (Homo sapiens) arose in Africa approximately 200,000 years ago, following approximately 6 million years of divergence from the shared great ape ancestors of Homo and chimpanzees (Pan troglodytes) [2]. Unlike chimpanzees, who remained confined to their home continent, approximately 60,000 years ago a single human population migrated out of Africa ultimately colonizing Eurasia, the Americas and Oceania. The long period since human-chimpanzee divergence combined with our subsequent worldwide spread has provided ample opportunity for evolution to differentially adapt the two species to novel environments.

Hepatitis C virus (HCV), an enveloped positive-sense RNA virus in the family Flaviviridae (genus Hepacivirus), is a blood-borne pathogen that is seemingly restricted to humans [3]. HCV currently infects an estimated 71 million people worldwide ( $\sim 1 \%$ of the human population) resulting in 399,000 deaths annually due its efficient ability to establish a chronic - typically life long - infection in the liver that can lead to fatal disease decades after primary infection in the absence of treatment. HCV is highly diverse with at least eight distinct 'genotypes', which can be over 30\% different genetically from each other [4].

While HCV likely originated in the 'Old World' - with genotypes endemic only in sub-Saharan Africa and Asia - a precise origin for the virus has not been identified [5]. Estimated timings for the expansion in HCV genomic diversity suggest an origin between hundreds of thousands to thousands of years ago. Thus, current evidence fails to distinguish between whether HCV has a 'recent' beginning in humans reflecting independent origins across Africa and Asia or a single more ancient one, with HCV leaving Africa at the time of human migration. This pattern of high genetic diversity in a single host species could be interpreted as evidence of either long-term coexistence of HCV with humans, and/or relatively efficient spillover into humans from an as yet unknown animal reservoir present in Africa and Asia; both scenarios indicate an intrinsic high level of HCV adaptation to humans. Of note, hepaciviral sequences have been found across a very diverse selection of mammals [6] with the greatest diversity found in bats and rodents, yet the closest relative to HCV is one found in horses and dogs but not primates.

To explore the virus' host tropism, HCV has only been inoculated into species belonging to the Euarchontoglires (rodents, rabbits, colugos, treeshrews and primates), for example: mice and woodchucks, treeshrews, and primates (e.g., apes, Old and New World monkeys) [7-10]. Only chimpanzees and, to a much lesser degree, treeshrews are permissive for HCV infection. Using in vivo and in vitro studies the lack of permissivity and susceptibility in lab mice has been mapped to blocked viral entry and immune evasion [7]. Intriguingly, while permissive for chronic $\mathrm{HCV}$ infection, rates of chronicity and associated disease differ in chimpanzees and treeshrews when compared with humans. Most notably, HCV-infected chimpanzees have reduced rates of chronicity [11], disease [8], treatment responses [12] and viral glycoprotein gene mutation rates indicative of enhanced immune pressure [13].

Future Medicine 
By exploiting variation of HCV infection outcomes, combined with comparisons of whole genome sequences or intraspecies genomic variation, research has started uncovering the specific host molecules that define species and individual differences in HCV chronicity and pathogenesis [14]. Downstream experimental analysis can provide additional functional evidence to support statistical associations. Initial studies demonstrated a significant role of interindividual genetic differences in humans in the cellular immune response, including the $\mathrm{T}$ cell and natural killer cell responses. More recently, genome-wide association studies in humans for HCV clearance revealed the impact of innate immune signaling genes. This work identified a common loss-of-function frameshift variant in the antiviral signaling protein gene IFNL4, associated with HCV clearance [15]. Somewhat counterintuitively for an antiviral protein, production of IFNL4 is associated with reduced clearance of acute HCV infection and reduced response to interferon alpha therapy, and has also been associated with reduced liver inflammation and disease [16]. A further common coding variant resulting from a substitution of proline at position 70 to serine (P70S) in IFNL4 was found to be associated with higher clearance rates than that of the 'wild-type' protein [17].

Sequencing of mammalian genomes and analysis of IFNL4 sequences showed that the gene has evolved under purifying selection across a limited - but broad - selection of mammal species [18]. The IFNL4 loss-of-function frameshift allele varies across human populations with its highest frequency in East Asia $(>90 \%)$ and lowest in sub-Saharan Africa (<40\%). Loss of IFNL4 has evolved under positive selection in African and Asian populations although the cause is unknown [18].

Intrigued by the role of IFNL4 variation in regulating HCV biology within humans and possibly between us and other species, we have shown recently that human IFNL4 has evolved markedly reduced antiviral activity, in comparison with IFNL4 from chimpanzees and to a lesser extent, from rhesus macaques [19]. This reduction in activity resulted from a single amino acid change arising during human evolution (glutamic acid 154 to lysine; E154K). E154 is almost 100\% conserved in mammalian IFNL4 sequences, with humans being the exception. Mechanistically, this negative-to-positive charge substitution impeded both protein secretion and potency likely through disrupting key intramolecular bonds. Furthermore, our in vitro observations correlated with reduced antiviral transcriptomic liver responses in humans compared with chimpanzees during HCV infection. The reduced activity is highly conserved in humans, with the E154 allele found in individuals with African descent, including African-Americans and 'pygmy' hunter-gatherers from Central Africa (but not other African hunter-gatherers). We also detected a very rare additional variant, L79F, in only one person from Sierra Leone in West Africa. Additionally, we were able to time the E154K event by identifying the variant in archaic human genomes (Neanderthal and Denisovan) and the human frameshift TT allele, suggesting a date of occurrence at some point between 6 million (post Homo-Pan divergence) and approximately 400,000 years ago (prior to human and Neanderthal/Denisovan divergence). The E154K mutation seems to be the initial activity-attenuating event during human evolution. This mutation likely occurred within Africa and was followed by rapid fixation in an early Homo ancestor before the frameshift and P70S mutations. What drove this rapid evolution of an antiviral signaling molecule with less activity is unknown.

Chimpanzees are considered a robust comparative model for HCV pathogenesis. Based on this, our work suggests that although $\mathrm{HCV}$ is still able to establish a chronic infection in chimpanzees, their higher IFNL4 activity compared with modern humans may favor the host and reduce the likelihood of chronicity and disease. Of note, alongside enhanced antiviral gene expression in HCV-infected chimpanzee livers, we observed enhanced levels of gene transcripts involved in T cell immune responses, which are critical for HCV clearance [19]. We thus propose that humans evolved enhanced susceptibility to HCV relatively early during evolution. However, subsequent evolution has led to both the seemingly maladaptive (E154K) and protective variants (IFNL4 loss-of-function, $\mathrm{P} 70 S$ ) coexisting in the same population resulting in a rich assemblage of variation in infection outcome. Therefore, comparative genomics have revealed that human-specific genetic changes occurring following human-chimpanzee divergence have both enhanced and reduced our immune defences to HCV.

Significant fundamental questions remain for even this most studied association: through what mechanism(s) do IFNL4 and its variants affect HCV chronicity and pathogenesis? As chimpanzees are no longer subjected to experimentation, and as the model would remain near impossible to genetically manipulate, this question and more can only be addressed through the development of novel experimental approaches, which captures unique aspects of HCV biology, such as chronicity and immunopathology. This could perhaps be achieved using other Hepacivirushost pairs, such as cows. Recently, it has been shown that IFNL4 drives HCV evolution within patients [20]. Thus, a broader understanding of the complex relationship between both host and pathogen genetic and phenotypic diversity should be pursued for HCV and other pathogens. 
Investigating the molecular determinants of host-pathogen interactions remains critical to understanding and manipulating the outcomes of microbial infection and pathogenesis. Recent advances in comparative and functional genomics from diverse host-pathogen pairs have begun to unravel the molecular mechanisms underlying outcome of infection. Given the significant global health burden resulting from its capacity to establish a chronic infection, studies on HCV are both needed and well-suited to continue providing critical insights into the workings of the human immune system and our evolution.

\section{Acknowledgements}

The authors would like to thank the McLauchlan lab for helpful discussions during the writing of this editorial.

\section{Financial \& competing interests disclosures}

Our work discussed in this editorial was funded by the UK Medical Research Council to J McLauchlan (https://mrc.ukri.org/; grant number: MC_UU_12014/1). The funders had no role in study design, data collection and analysis, decision to publish or preparation of the manuscript. The authors have no other relevant affiliations or financial involvement with any organization or entity with a financial interest in or financial conflict with the subject matter or materials discussed in the manuscript apart from those disclosed.

No writing assistance was utilized in the production of this manuscript.

\section{Open access}

This work is licensed under the Creative Commons Attribution 4.0 License. To view a copy of this license, visit http://creativecommons.org/licenses/by/4.0/

\section{References}

1. Fan S, Hansen MEB, Lo Y et al. Going global by adapting local: a review of recent human adaptation. Science 354(6308), 54-59 (2016).

2. Quach H, Quintana-Murci L. Living in an adaptive world: genomic dissection of the genus Homo and its immune response. J. Exp. Med. 214(4), 877-894 (2017).

3. Simmonds P, Becher P, Bukh J et al. ICTV virus taxonomy profile: Flaviviridae. J. Gen. Virol. 98(1), 2-3 (2017).

4. Smith DB, Bukh J, Kuiken C et al. Expanded classification of hepatitis C virus into 7 genotypes and 67 subtypes: updated criteria and genotype assignment web resource. Hepatology 59(1), 318-327 (2014).

5. Simmonds P. The origin of hepatitis C virus. Curr. Top. Microbiol. Immunol. 369, 1-15 (2013).

6. Pybus OG, Thézé J. Hepacivirus cross-species transmission and the origins of the hepatitis C virus. Curr. Opin. Virol. 16, 1-7 (2016).

7. Dorner M, Horwitz JA, Donovan BM et al. Completion of the entire hepatitis $\mathrm{C}$ virus life cycle in genetically humanized mice. Nature 501(7466), 237-241 (2013).

8. Walker CM. Comparative features of hepatitis C virus infection in humans and chimpanzees. Semin. Immunopathol. 19(1), 85-98 (1997).

9. Abe K, Kurata T, Teramoto Y et al. Lack of susceptibility of various primates and woodchucks to hepatitis C virus. J. Med. Primatol. 22(7-8), 433-434 (1993).

10. Feng Y, Feng Y-M, Lu C et al. Tree shrew, a potential animal model for hepatitis C, supports the infection and replication of HCV in vitro and in vivo. J. Gen. Virol. 98(8), 2069-2078 (2017).

11. Bassett SE, Brasky KM, Lanford RE. Analysis of hepatitis C virus-inoculated chimpanzees reveals unexpected clinical profiles. J. Virol. 72(4), 2589-2599 (1998).

12. Lanford RE, Guerra B, Lee $\mathrm{H}$ et al. Genomic response to interferon-alpha in chimpanzees: implications of rapid downregulation for hepatitis C kinetics. Hepatology. 43(5), 961-972 (2006).

13. Ray SC, Mao Q, Lanford RE et al. Hypervariable region 1 sequence stability during hepatitis $\mathrm{C}$ virus replication in chimpanzees. J. Virol. 74(7), 3058-3066 (2000).

14. Gauthiez E, Habfast-Robertson I, Rüeger S et al. A systematic review and meta-analysis of HCV clearance. Liver Int. 37(10), 1431-1445 (2017).

15. Prokunina-Olsson L, Muchmore B, Tang W et al. A variant upstream of IFNL3 (IL28B) creating a new interferon gene IFNL4 is associated with impaired clearance of hepatitis C virus. Nat. Genet. 45(2), 164-171 (2013).

16. Eslam M, Hashem AM, Leung R et al. Interferon- $\lambda$ rs 12979860 genotype and liver fibrosis in viral and non-viral chronic liver disease. Nat. Commun. 6(1), 6422 (2015).

17. Terczyńska-Dyla E, Bibert S, Duong FHT et al. Reduced IFN $\lambda 4$ activity is associated with improved HCV clearance and reduced expression of interferon-stimulated genes. Nat. Commun. 5, 5699 (2014).

18. Key FM, Peter B, Dennis MY et al. Selection on a variant associated with improved viral clearance drives local, adaptive pseudogenization of interferon lambda 4 (IFNL4). PLoS Genet. 10(10), e1004681 (2014). 
19. Bamford CGG, Aranday-Cortes E, Filipe IC et al. A polymorphic residue that attenuates the antiviral potential of interferon lambda 4 in hominid lineages. PLoS Pathog. 14(10), e1007307 (2018).

20. Ansari MA, Pedergnana V, Ip CL et al. Genome-to-genome analysis highlights the effect of the human innate and adaptive immune systems on the hepatitis C virus. Nat. Genet. 49(5), 666-673 (2017). 